\title{
Calidad de vida en mujeres con cáncer de mama que acuden al Departamento de Oncología. Hospital Nacional Daniel Alcides Carrión. Callao - 2018
}

Paulo Olivares-Taipe* 1,c,d; José Aguilar-Saenz 1,b,s; Rolando Adrianzen-Tantachuco 2,a; José Revilla-López ${ }^{3, a}$; Allyn ZavaletaPesantes ${ }^{4, a, e}$; Giovanini Martinez-Asmad ${ }^{4, e, f}$; Alejandro Héctor Huapaya Cabrera 1,a

RESUMEN

Objetivo: Determinar la calidad de vida en mujeres con cáncer de mama que acuden al Departamento de Oncología en el Hospital Nacional Daniel Alcides Carrión (Callao).

Materiales y métodos: El estudio fue de tipo básico, diseño observacional, nivel descriptivo, prospectivo y de corte transversal. Las unidades muestrales fueron 100 pacientes con cáncer de mama, en el periodo correspondiente a mayo y julio del 2018. El instrumento de recolección de datos es un cuestionario con 34 ítems.

Resultados: La mayoría de mujeres con esta enfermedad se ubican en el nivel regular ( 93 \%), en la dimensión física la mayoría de casos están en el nivel regular (98\%), la dimensión psicológica también se ubica en el nivel regular (87 \%), y en la dimensión social en el nivel malo (77\%).

Conclusiones: Las características de las mujeres con esta enfermedad son dificultades en diferentes partes del cuerpo; en la dimensión psicológica presentan una calidad de vida poco saludable con intranquilidad y ansiedad; en la dimensión social, la calidad de vida es de nivel malo, lo que describe que muchas mujeres que padecen el mal participan muy poco de las reuniones sociales, visitas de recreación y manifiestan un alejamiento de sus amistades y familiares.

Palabras clave: Calidad de vida; Cáncer de mama; Salud Pública; Oncología (Fuente: DeCS BIREME).

\section{Quality of life in women with breast cancer who visit the Oncology Department of the Hospital Nacional Daniel Alcides Carrión. Callao - 2018}

ABSTRACT

Objective: To determine the quality of life in women with breast cancer who visit the Oncology Department of the Hospital Nacional Daniel Alcides Carrión (Callao).

Materials and methods: This study had a basic, observational, descriptive, prospective and cross-sectional design. The sample consisted of 100 patients with breast cancer who visited the hospital between May and July 2018. The data collection instrument was a 34-item questionnaire.

Results: Most women with this disease had a fair quality of life (93\%). In the physical and psychological dimensions, most of them also presented a fair quality of life ( $98 \%$ and $87 \%$, respectively) and, in the social dimension, they showed a poor quality of life $(77 \%)$.

Conclusions: These women experienced problems in different parts of their body. In the psychological dimension, they presented an unhealthy quality of life generating a sense of uneasiness and anxiety. In the social dimension, they also presented a poor quality of life resulting in little participation in social gatherings and recreational visits, and a rift from friends and family.

Keywords: Quality of life; Breast neoplasms; Public health; Medical oncology (Source: MeSH NLM).

1 Universidad Privada Arzobispo Loayza, Oficina de Investigación. Lima, Perú.

2 Hospital Nacional Daniel Alcides Carrión, Área de cirugía. Callao, Perú.

3 Hospital Nacional Daniel Alcides Carrión, Departamento de Oncología. Callao, Perú.

4 Universidad Privada Arzobispo Loayza, Escuela profesional de Obstetricia. Lima, Perú.

5 Universidad Privada Arzobispo Loayza, Secretaría General. Lima, Perú.

a Médico; b Maestro en Problemas de Aprendizaje; c Investigacion Operativa; d Maestro en Docencia Universitaria; e Doctor(a) en Administración de la Educación; f Obstetra; g Educador; h Maestro en gerencia de servicios de salud.

* Autor corresponsal 
Paulo Olivares-Taipe; José Aguilar-Saenz; Rolando Adrianzen-Tantachuco; José Revilla-López; Allyn Zavaleta-Pesantes; Giovanini Martinez-Asmad; Alejandro Héctor Huapaya Cabrera

\section{INTRODUCCIÓN}

El cáncer es un problema de salud pública en todo el mundo. Anualmente, se diagnostican, aproximadamente, 12,7 millones de casos nuevos, y para el 2030, serán 21,3 millones de casos nuevos (1). En 2018, en Estados Unidos se estimó unas 252710 mujeres con cáncer de mama invasivo, y 63410 mujeres con cáncer de mama in situ ${ }^{(2)}$. La Organización Panamericana de la Salud señaló que, en América, las muertes por esta enfermedad pasarán de 1,2 millones, en el 2008, a 2,1 millones al $2030^{(3)}$.

De acuerdo a la Organización Mundial de la Salud, el cáncer de mama es el padecimiento más común entre las mujeres de todos los países. En el 2015, la enfermedad se encontraba entre los 5 tipos de cáncer que causaron el mayor número de fallecimientos (571 000 muertes) ${ }^{(4)}$. En América Latina, el cáncer de mama fue la segunda causa de muerte, y es el que más vidas cobra en las mujeres latinoamericanas: del 5 al $9 \%$ de las muertes entre los 20 y 59 años de edad. En 2012, más de 408200 mujeres fueron diagnosticadas con cáncer de mama en América, y se calcula que 92000 fallecieron por la enfermedad. En este grupo, casi la mitad de las muertes (47\%), se registraron en América Latina y el Caribe. Adicionalmente, se estima que el número de mujeres a las que se les diagnosticará este tipo de cáncer en América aumentará en 46 \% para el $2030{ }^{(5)}$. Esta situacion tiene un alcance en el Perú; los registros de hospitales con capacidad diagnóstica indican, respecto al cáncer de mama, en pacientes de sexo femenino, una predominancia del $14,3 \%$ entre enero y diciembre del 2017. Otro dato importante es que el 63,8 \% de los casos diagnosticados en los diferentes tipos de cáncer presentan síntomas clínicos, lo que indica que las dos terceras partes de los casos fueron diagnosticados tardíamente ${ }^{(6)}$.

Por esto, es necesario implementar estrategias de cuidado que involucren tanto a la mujer como a la familia, para lograr una mejor calidad de vida durante el tratamiento y la recuperación ${ }^{(7)}$. El cáncer de mama un proceso oncológico, por lo que se espera que las pacientes sientan ansiedad, incertidumbre y tristeza; lo que implica que se debe aprender a manejar los cambios en la vida con una actitud positiva para enfrentar el temor y el impacto del diagnóstico en la vida personal. Este hecho pone de manifiesto el valor de la prevención y del conocimiento de la calidad de vida en estas pacientes.

En Perú, un estudio realizado en el Instituto Nacional de Enfermedades Neoplásicas indicó que $18 \%$ de pacientes oncológicos perciben la calidad de vida en un nivel bueno, el $60 \%$ reporta un nivel regular, y describe su experiencia como poco funcional en el ámbito personal, $22 \%$ manifiesta que no es saludable ya que su vida está afectada en los ámbitos social, físico y mental. Esta investigación concluye que la calidad de vida en las pacientes con la enfermedad es de nivel regular. Se observaron, según los resultados, características de dolor, debilidad, agotamiento, irritación, asimismo, tienen problemas para recordar y conciben que el tratamiento afectó su vida social. También se determinó que las tres dimensiones (física, psicológica y social) presentan un deterioro en la calidad de vida en un nivel regular ${ }^{(8)}$.

Lo anterior implica componente subjetivo de la percepción de bienestar y satisfacción, relacionado a los distintos aspectos de la vida. Por ello, la interpretación que haga la persona será percibida desde la calidad de vida que disfrute, y según los cambios que experimente, y de acuerdo a la actitud que asuma hacia dicha enfermedad.

La Organización Mundial de la Salud (OMS) define la calidad de vida (CV) como el entorno cultural caracterizado por las vivencias, opiniones, expectativas, y el sufrimiento de la persona que padece una enfermedad (9). En salud, es imprescindible abordar la enfermedad con el concepto de CV definida como la valoración que la mujer con cáncer de mama tiene sobre su bienestar global, es decir, el estado de salud, el tratamiento y las consecuencias de la enfermedad (10). Por lo general se mide la CV a través de cuestionarios autoadministrados, y el constructo se constituye en un indicador de la calidad del cuidado que la paciente.

Las diferentes observaciones de la medición de la CV, se han caracterizado por sus múltiples cuestionamientos y limitaciones, ya que es un concepto cambiante que no tiene correlato entre el estado de salud y la calidad de vida. Sin embargo, a pesar de ser tan variable, se relaciona con intervenciones cuyo propósito es extender la vida y mejorar la calidad de la misma. Por otro lado, encontramos dos sesgos que deben considerarse al evalualar la CV: primero, los pacientes más graves que tienen poca disposición a los cuestionario o encuestas, y segundo, la influencia del aspecto afectivo y su apreciación en la autovaloración de CV ${ }^{(11,12)}$. No obstante, se concuerda que sus ventajas son mayores que sus desventajas ${ }^{(13,14)}$.

El cáncer de mama es una enfermedad con efectos en la vida personal, que se agudizan cuando se encuentran en estadios avanzados de la enfermedad. Además, es un problema que tiene incidencias psicológicas, emocionales y sociales graves, que requieren un abordaje profesional y holístico ${ }^{(15,16)}$.

Por ello, las pacientes con cáncer de mama están en un momento de toma de decisiones, referidas al hecho de afrontar cambios en su estilo de vida. En vista de estas consideraciones, la adaptación a la enfermedad favorecerá la calidad de vida de estas pacientes oncológicas. Por ello es necesario, establecer en qué medida las mujeres que padecen esta enfermedad son afectadas en su calidad de vida personal y social ${ }^{(17)}$. Por lo tanto, el objetivo principal de la 
investigación es determinar la calidad de vida en mujeres con cáncer de mama que acuden al Departamento de Oncología en el Hospital Nacional Daniel Alcides Carrión (Callao).

\section{MATERIALES Y MÉTODOS}

\section{Diseño y población de estudio}

Se realizó un estudio de tipo básico, diseño observacional, nivel descriptivo, prospectivo y de corte transversal, que describe la calidad de vida, y su relación con las variables sociodemográficas, en mujeres que padecen cáncer de mama ${ }^{(18-20)}$.

La población estuvo conformada por mujeres con cáncer de mama que asistieron al servicio oncológico durante los meses mayo a julio del 2018. Se tomó una muestra de 100 pacientes del Hospital Nacional Daniel Alcides Carrión en edades comprendidas entre los 22 y 94 años, seleccionadas de manera no probabilística. Las pacientes fueron contactadas a través del jefe de la Unidad Oncológica, participaron de manera voluntaria y firmaron el respectivo consentimiento informado.

\section{Variables y mediciones}

Las pacientes del servicio oncológico se seleccionaron por razones demográficas y clínicas según el registro de historias del hospital. Las variables analizadas fueron la calidad de vida (CV), las variables sociodemográficas (edad, estado civil, grado de instrucción, situación laboral y situación económica) y las variables intervinientes (fase y tratamiento del paciente).

El instrumento de recolección de datos fue un cuestionario elaborado por Arteaga Llacza ${ }^{(8)}$, validado por especialistas en oncología y metodología del INEN, estructurado en 34 preguntas y organizado en las dimensiones física (14 ítems), psicológica (11 ítems) y social (9 ítems) con opciones de respuesta ordinales.

Es importante establecer que la enfermedad tiene implicancias en la vida de las pacientes que ya presentan sintomatología clínica, debido al diagnóstico tardío en muchos de los $\operatorname{casos}^{(21)}$. Se prescindieron de las pacientes con síndromes mieoloproliferativos o mielodisplásicos, neoplasias benignas, gestantes o que fueron tratadas previamente en otra institución. La calidad de vida se define como la valoración subjetiva del paciente sobre su bienestar global relacionado a su estado general, a las consecuencias y los tratamientos de la enfermedad (10).

Cabe destacar que la variable y sus dimensiones son percibidas por las pacientes en las distintas dimensiones a estudiar. Por tales motivos, la dimensión física se percibe desde su estado físico, respecto a los síntomas y los efectos adversos del tratamiento. La dimensión psicológica se evalua a partir del estado cognitivo y afectivo, y la dimensión social describe las relaciones interpersonales y los roles sociales relacionados al apoyo familiar, a la relación médico-paciente y el desempeño laboral (17).

\section{Análisis estadístico}

Se empleó el programa estadístico SPSS versión 24.0. Se utilizó la estadística descriptiva y se consideró el valor promedio, la desviación estándar (Desv. Standar) y el valor máximo y mínimo para las variables cuantitativas; también se realizaron estimaciones para las variables nominales y se obtuvieron los valores de frecuencia y porcentaje. Los resultados se presentaron en tablas que consignaban el número total de pacientes.

\section{Consideraciones éticas}

El Comité de Ética del Hospital Nacional Daniel Alcides Carrión aprobó el proyecto de investigación en el marco de la Declaración de Helsinki ${ }^{(22)}$. La información analizada fue de uso exclusivo del grupo de investigadores.

\section{RESULTADOS}

Se encuestó a un total de 100 pacientes con de cáncer de mama, con una edad promedio de 53 años $( \pm 14,6)$, y que fluctuaron entre los 22 a 94 años. La mayor proporción de mujeres con cáncer de mama son solteras (35\%), el grado de instrucción en la mayoría de pacientes es educación secundaria (58\%), $85 \%$ son desempleadas, y la situación económica es, mayoritariamente, regular (79 \%) (Tabla 1).

Figura 1. Distribución de las pacientes con cáncer de mama que acuden al departamento de Oncología del hospital Nacional Daniel Alcides Carrión del Callao según datos sociodemográficos

\begin{tabular}{|c|c|c|c|c|}
\hline \multirow[t]{2}{*}{ Calidad } & $\begin{array}{l}\text { Máximo } \\
94\end{array}$ & $\underset{22}{\text { Mínimo }}$ & $\begin{array}{c}\text { Desv. Standar } \\
14,6\end{array}$ & $\begin{array}{c}\text { Media } \\
53\end{array}$ \\
\hline & \multicolumn{2}{|c|}{ Frecuencia } & \multicolumn{2}{|c|}{ Porcentaje (\%) } \\
\hline \multicolumn{5}{|c|}{ Estado civil } \\
\hline Soltera & & & & \\
\hline Casada & & & & \\
\hline
\end{tabular}


Paulo Olivares-Taipe; José Aguilar-Saenz; Rolando Adrianzen-Tantachuco; José Revilla-López; Allyn Zavaleta-Pesantes; Giovanini Martinez-Asmad; Alejandro Héctor Huapaya Cabrera

\begin{tabular}{|c|c|c|}
\hline \multirow[t]{2}{*}{ Calidad } & $\underset{94}{\text { Máximo }}$ & $\begin{array}{c}\text { Desv. Standar } \\
14,6\end{array}$ \\
\hline & Frecuencia & Porcentaje (\%) \\
\hline Viuda & 9 & $9,0 \%$ \\
\hline Divorciada & 2 & $2,0 \%$ \\
\hline Conviviente & 26 & $26,0 \%$ \\
\hline \multicolumn{3}{|c|}{ Grado de instrucción } \\
\hline Primaria & 17 & $17,0 \%$ \\
\hline Secundaria & 58 & $58,0 \%$ \\
\hline Superior & 25 & $25,0 \%$ \\
\hline \multicolumn{3}{|l|}{ Situación laboral } \\
\hline Empleada & 15 & $15,0 \%$ \\
\hline Desempleada & 85 & $85,0 \%$ \\
\hline \multicolumn{3}{|c|}{ Situación económica } \\
\hline Buena & 5 & $5,0 \%$ \\
\hline Regular & 79 & $79,0 \%$ \\
\hline Mala & 16 & $16,0 \%$ \\
\hline
\end{tabular}

Las mujeres con cáncer de mama se encuentran mayoritariamente en la fase II (52\%); la mayoría (68\%) recibe quimioterapia, $78 \%$ no reciben radioterapia y $75 \%$ no recibe hormonoterapia. El $61 \%$ ha recibido tratamiento quirúrgico, y $100 \%$ indicó que no recibieron tratamiento biológico (Tabla 2).

Tabla 2. Fase y tratamiento en la que se encuentra la paciente

\begin{tabular}{|ccc|}
\hline & Frecuencia & Porcentaje (\%) \\
\hline Etapa & 12 & $12,0 \%$ \\
\hline Fase I & 52 & $52,0 \%$ \\
\hline Fase II & 26 & $26,0 \%$ \\
\hline Fase III & 10 & $10,0 \%$ \\
\hline Fase IV & & \\
\hline Quimioterapia & 68 & $68,0 \%$ \\
\hline Sí & 32 & $32,0 \%$ \\
\hline No & 22 & $22,0 \%$ \\
\hline Radioterapia & 78 & $78,0 \%$ \\
\hline Sí & & \\
\hline No & 25 & $25,0 \%$ \\
\hline Hormonoterapia & 75 & $75,0 \%$ \\
\hline Sí & & \\
\hline No & 61 & $61,0 \%$ \\
\hline Quirúrgico & 39 & $39,0 \%$ \\
\hline Sí & & $0 \%$ \\
\hline No & 0 & $100,0 \%$ \\
\hline Biológico & 100 & \\
\hline Sí & & \\
\hline No & & \\
\hline & & \\
\hline
\end{tabular}


Según el nivel de estado de la paciente y de acuerdo a las dimensiones, la percepción de la calidad de vida es, en general , regular (93\%); en la dimensión física es $98 \%$; en la dimensión psicológica, $87 \%$, y en la dimensión social, se percibe como mala (77 \%) (Tabla 3$)$.

Tabla 3. Niveles de la variable y sus dimensiones

\begin{tabular}{|ccc|}
\hline & Frecuencia & Porcentaje (\%) \\
\hline General & & \\
\hline Regular & 93 & $93,0 \%$ \\
\hline Malo & 7 & $7,0 \%$ \\
\hline Dimensión física & & \\
\hline Regular & 98 & $98,0 \%$ \\
\hline Malo & 2 & $2,0 \%$ \\
\hline Dimensión psicológica & & \\
\hline Regular & 87 & $87,0 \%$ \\
\hline Malo & 13 & $13,0 \%$ \\
\hline Dimensión social & & \\
\hline Regular & 23 & $23,0 \%$ \\
\hline Malo & 77 & $77,0 \%$ \\
\hline
\end{tabular}

La calidad de vida en general, y las dimensiones física y psicológica en los estadios clínicos I al IV, están en nivel regular. En la dimensión social, en las etapas I al IV, la mayor tendencia porcentual se ubica en el nivel malo.
Por otro lado, en relación al tratamiento quirúrgico, quimioterapia y radioterapia, está, igualmente, en el plano regular (Tabla 4).

Figura 4. Niveles de la variable y sus dimensiones, según estadio clínico y de tratamiento

\begin{tabular}{|c|c|c|c|c|c|c|c|}
\hline Calidad & & & & & Estadio clínico & Quimioterapia & Radioterapia \\
\hline General & I & II & III & IV & Sí & Sí & Sí \\
\hline Regular & $\begin{array}{c}11 \\
(100 \%)\end{array}$ & $\begin{array}{c}47 \\
(90,4 \%)\end{array}$ & $\begin{array}{c}25 \\
(96,2 \%)\end{array}$ & $\begin{array}{c}9 \\
(90 \%)\end{array}$ & $\begin{array}{c}57 \\
(93,4 \%)\end{array}$ & $\begin{array}{c}63 \\
(92,6 \%)\end{array}$ & $\begin{array}{c}21 \\
(95,5 \%)\end{array}$ \\
\hline Mala & $\begin{array}{c}0 \\
(0 \%)\end{array}$ & $\begin{array}{c}5 \\
(9,6 \%)\end{array}$ & $\begin{array}{c}1 \\
(3,8 \%)\end{array}$ & $\begin{array}{c}1 \\
(10 \%)\end{array}$ & $\begin{array}{c}4 \\
(6,6 \%)\end{array}$ & $\begin{array}{c}5 \\
(7,4 \%)\end{array}$ & $\begin{array}{c}1 \\
(4,5 \%)\end{array}$ \\
\hline \multicolumn{8}{|l|}{ Física } \\
\hline Regular & $\begin{array}{c}11 \\
(100 \%)\end{array}$ & $\begin{array}{c}51 \\
(98,1 \%)\end{array}$ & $\begin{array}{c}26 \\
(100 \%)\end{array}$ & $\begin{array}{c}9 \\
(90 \%)\end{array}$ & $\begin{array}{c}60 \\
(98,4 \%)\end{array}$ & $\begin{array}{c}66 \\
(97,1 \%)\end{array}$ & $\begin{array}{c}21 \\
(95,5 \%)\end{array}$ \\
\hline Mala & $\begin{array}{c}0 \\
(0 \%)\end{array}$ & $\begin{array}{c}1 \\
(1,9 \%)\end{array}$ & $\begin{array}{c}0 \\
(0 \%)\end{array}$ & $\begin{array}{c}1 \\
(10 \%)\end{array}$ & $\begin{array}{c}1 \\
(1,6 \%)\end{array}$ & $\begin{array}{c}2 \\
(2,9 \%)\end{array}$ & $\begin{array}{c}1 \\
(4,5 \%)\end{array}$ \\
\hline \multicolumn{8}{|c|}{ Psicológica } \\
\hline Regular & $\begin{array}{c}10 \\
(90,9 \%)\end{array}$ & $\begin{array}{c}45 \\
(86,5 \%)\end{array}$ & $\begin{array}{c}24 \\
(92,3 \%)\end{array}$ & $\begin{array}{c}7 \\
(70 \%)\end{array}$ & $\begin{array}{c}54 \\
(88,5 \%)\end{array}$ & $\begin{array}{c}62 \\
(91,2 \%)\end{array}$ & $\begin{array}{c}20 \\
(90,9 \%)\end{array}$ \\
\hline Mala & $\begin{array}{c}1 \\
(9,1 \%)\end{array}$ & $\begin{array}{c}7 \\
(13,5 \%)\end{array}$ & $\begin{array}{c}2 \\
(7,7 \%)\end{array}$ & $\begin{array}{c}3 \\
(30 \%)\end{array}$ & $\begin{array}{c}7 \\
(11,5 \%)\end{array}$ & $\begin{array}{c}6 \\
(8,8 \%)\end{array}$ & $\begin{array}{c}2 \\
(9,1 \%)\end{array}$ \\
\hline
\end{tabular}


Paulo Olivares-Taipe; José Aguilar-Saenz; Rolando Adrianzen-Tantachuco; José Revilla-López; Allyn Zavaleta-Pesantes; Giovanini Martinez-Asmad; Alejandro Héctor Huapaya Cabrera

\begin{tabular}{cccccccc|} 
Calidad & \multicolumn{1}{c}{} & & Estadio clínico & Quimioterapia & Radioterapia \\
\hline Social & & & & & & \\
Regular & 2 & 8 & $10(38,5 \%)$ & 2 & 10 & 12 & 6 \\
& $(18,2 \%)$ & $(15,4 \%)$ & & $(20 \%)$ & $(16,4 \%)$ & $(17,6 \%)$ & $(27,3 \%)$ \\
Mala & 9 & 44 & $16(61,5 \%)$ & 8 & 51 & 56 & 16 \\
& $(81,8 \%)$ & $(84,6 \%)$ & & $(80 \%)$ & $(83,6 \%)$ & $(82,4 \%)$ & $(72,7 \%)$ \\
& & & & & & & \\
\hline
\end{tabular}

\section{DISCUSIÓN}

Los principales hallazgos en la investigación responden a los objetivos planteados inicialmente. Encontramos que las mujeres con cáncer de mama tienen una calidad de vida predominantemente regular tanto en el aspecto físico y psicológico. En la dimensión social, se encuentran en un nivel malo. Se demostró que $52 \%$ de las participantes están en la fase II de la enfermedad. La mayoría son solteras con una situación económica regular. Los resultados indican que el cáncer de mama afecta la calidad de vida de la pacientes. Estos hallazgos son similares a los presentados en un estudio del INEN que reportó la calidad de vida del paciente oncológico en un nivel regular ${ }^{(8)}$.

Un hallazgo relevante del estudio fue que la calidad de vida se ubica en un nivel regular en un $93 \%$, este resultado se refiere a un hábito o forma de vida con actitudes cotidianas poco satisfactorias que afectan la salud física, mental y social de la paciente. Esta nueva forma de vida conlleva una baja satisfacción de las necesidades humanas para alcanzar la calidad de vida. Por lo tanto, la percepción que tienen respecto a su estado de salud, asociado a las relaciones y vínculos con la familia, los pares y amigos se encuentran afectados por la enfermedad.

El cáncer de mama implica una serie de emociones relacionadas con el tratamiento y con efectos en la imagen corporal. Es importante mencionar que no todos los casos de esta enfermedad son iguales, que se diferencian por las fases en que se encuentran (fase II) ${ }^{(3)}$. También se destaca que, en el caso de tratamiento quirúrgico, este impacta fuertemente en la estructura física y funcional de las mujeres. Por lo tanto, la calidad de vida se ve afectada, no solo por la enfermedad, sino también por el tipo de secuela que, visualmente, no es agradable ${ }^{(23)}$. Nuestro estudio permite conocer un campo poco estudiado; sin embargo, se requiere mayor investigación en relación a la calidad de vida de las pacientes con cáncer de mama.

Concluimos que las pacientes con cáncer de mama perciben la calidad de vida en un nivel regular en los ámbitos general (93\%), físico (98\%) y psicológico (87\%), mientras que lo consideran en un nivel malo en el aspecto social $(77 \%)$.

\section{REFERENCIAS BIBLIOGRÁFICAS}

1. The Lancet Oncology Comisión. La planificación del control del cáncer en América Latina y el Caribe. Lancet Oncol. 2013; 14: 1-52.

2. American Society of Clinical Oncology (ASCO). Cáncer de mama: estadísticas [Internet]. 2017. Disponible en: https:// www.cancer.net/es/tipos-de-c\%C3\%A1ncer/c\%C3\%A1ncer-demama/estad\%C3\%ADsticas.

3. Organización Panamericana de la Salud (OPS). El cáncer en la región de las Américas [Internet]. 2012. Disponible en: https: / / www. paho.org/hq/dmdocuments/2014/OPS-NotaInformativa-Cancer-2014.pdf

4. Organización Mundial de la Salud (OMS). Cáncer [Internet]. 2017. Disponible en: http://www.who.int/mediacentre/ factsheets/fs297/es/

5. Chubb.lat. El cáncer de mama en Latinoamérica [Internet]. 2017. Disponible en: https://chubb.lat/enlace/ es/2017/04/18/el-cancer-de-mama-en-latinoamerica

6. Centro Nacional de Epidemiología, Prevención y Control de Enfermedades. Boletín epidemiológico del Perú SE 19-2018. Lima: Ministerio de Salud; 2018. Disponible en: https://www. dge.gob.pe/portal/docs/vigilancia/boletines/2018/19.pdf

7. Aquino Gargiulo C, Simões Cardoso de Melo MC, De Oliveira Salimena AM, Freitas Bara VM, De Oliveira Souza IE. Vivenciando o cotidiano do cuidado na percepcão de enfermeiras oncológicas. Texto Contexto Enferm. 2007; 16(4): 696-702.

8. Arteaga Llacza PS. Calidad de vida del paciente oncológico que participa en estudios clínicos de investigación en el Instituto Nacional de Enfermedades Neoplásicas 2009 [Tesis]. Lima: Universidad Nacional Mayor de San Marcos. Facultad de Enfermería; 2010.

9. American Cancer Society. Cancer pain [Internet]. Disponible en: https://www.cancer.org/treatment/treatments-andside-effects/physical-side-effects/ pain. html.

10. Urzúa MA. Calidad de vida relacionada con la salud: elementos conceptuales. Rev Med Chile. 2010; 138(3): 358-65.

11. Grau J. Calidad de vida y salud: problemas actuales en su investigación. Boletín Latinoamericano de psicología de la salud. 2009.

12. Gill TM, Feinstein AR. A critical appraisal of the quality of quality-of-life measurements. JAMA. 1994; 272(8): 619-26.

13. Arrarás JI, Martínez M, Manterota A, Laínez N. La evaluación 
Calidad de vida en mujeres con cáncer de mama que acuden al Departamento de Oncología.

Hospital Nacional Daniel Alcides Carrión. Callao - 2018

de la calidad de vida del paciente oncológico. El grupo de calidad de vida de la EORTC. Psicooncología. 2004; 1(1): $87-$ 98.

14. Perry S, Kowalski TL, Chang CH. Quality of life assessment in women with breast cancer: benefits, acceptability and utilization. Health Qual Life Outcomes 2007; 5: 24.

15. Cruickshank S, Kennedy C, Lockhart K, Dosser I, Dallas L. Specialist breast care nurses for supportive care of women with breast cancer. Cochrane Database Syst Rev. 2008; (1): CD005634.

16. Vivar CG. Calidad de vida en supervivientes a largo plazo de cáncer de mama. Un área olvidada en la investigación enfermera española. Index Enferm. 2005; 14(50): 25-9.

17. Velázquez Leyva E, Ruiz Paloalto ML, Padilla Raygoza N, Favela Ocaño MA, Barragán Hernández O. Ansiedad y calidad de vida en la mujer con cáncer de mama. EPISTEMUS. 2015; 9: $14-23$.

18. Valderrama Mendoza S. Pasos para elaborar proyectos de investigación científica. 2a ed. Lima: San Marcos E. I. R. L.; 2015.

19. Hernández Sampieri R, Fernández Collado C, Baptista Lucio P. Metodología de la investigación. 5a ed. México D. F.: Mc Graw-Hill; 2014.

20. De Canales FH, De Alvarado EL, Pineda EB, Organización Panamericana de la Salud, Organización Mundial de la Salud. Metodología de la investigación. Manual para el desarrollo de personal de salud. 6a ed. México: Limusa; 2014.

21. Salas Zapata C, Grisales Romero H. Calidad de vida y factores asociados en mujeres con cáncer de mama en Antioquia, Colombia. Rev Panam Salud Publica. 2010; 28(1): 9-18.

22. Asocación Médica Mundial (AMM). Declaración de Helsinki de la $A M M$ - Principios Éticos para las investigaciones médicas en seres humanos [Internet]. Francia; 2018. Disponible en: https://www.wma.net/es/policies-post/ declaracion-de-helsinki-de-la-amm-principios-eticos-paralas-investigaciones-medicas-en-seres-humanos/

23. Martínez-Basurto AE, Lozano-Arrazola A, Rodríguez-Velázquez AL, Galindo-Vázquez O, Alvarado-Aguilar S. Impacto psicológico del cáncer de mama y la mastectomía. Gaceta Mexicana de Oncología. 2014; 13(1): 53-8.

Fuentes de financiamiento:

Este artículo ha sido financiado por los autores.

Conflictos de interés:

Los autores declaran no tener ningún conflicto los autores.

\section{Correspondencia:}

Paulo Cesar Olivares-Taipe

Dirección: Urb. Las Colinas Mz S lote 13. Callao, Perú. Teléfono: 990293355

Correo electrónico: paulocesarolivarestaipe@gmail.com

\section{Recibido: 03 de noviembre de 2018 \\ Evaluado: 25 de marzo de 2019 Aprobado: 01 de mayo de 2019.}

(c) La revista. Publicado por Universidad de San Martín de Porres, Perú. (c) в bajo términos de Licencia Creative Commons Atribución 4.0 Internacional. (http://creativecommons.org/licenses/by/4.0/)

\section{ORCID iDs}

Paulo César Olivares-Taipe José Aguilar-Saenz Rolando adrianzen-Tantachuco José Revilla-López Allyn Zavaleta-Pesantes Giovanini Martinez-Asmad Alejandro Huapaya-Cabrera https://orcid.org/0000-0001-9989-0709 https://orcid.org/0000-0002-7338-3749 https://orcid.org/0000-0001-9983-108X https://orcid.org/0000-0002-7594-0211 https: //orcid.org/0000-0002-0188-3875 https://orcid.org/0000-0002-3151-2557 https://orcid.org/0000-0001-8855-0805 